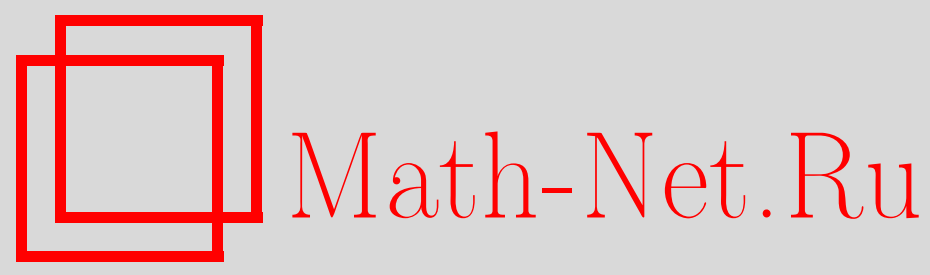

П. А. Крутицкий, Первая начально-краевая задача для одного уравнения составного типа в трехмерной многосвязной области, Матем. заметки, 2000, том 68, выпуск 2, 249-265

DOI: https://doi.org/10.4213/mzm942

Использование Общероссийского математического портала Math-Net.Ru подразумевает, что вы прочитали и согласны с пользовательским соглашением http://www . mathnet.ru/rus/agreement

Параметры загрузки:

IP : 34.229 .108 .108

26 апреля 2023 г., $16: 35: 30$

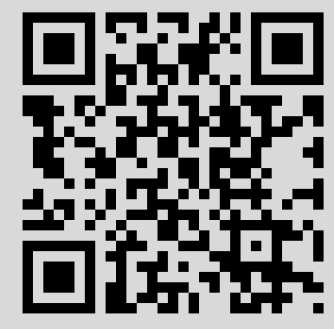




\section{ПЕРВАЯ НАЧАЛЬНО-КРАЕВАЯ ЗАДАЧА ДЛЯ ОДНОГО УРАВНЕНИЯ СОСТАВНОГО ТИПА В ТРЕХМЕРНОЙ МНОГОСВЯЗНОЙ ОБЛАСТИ}

\section{П. А. Крутицкий}

Метод граничных интегральных уравнений применяется к первой начально-краевой задаче для уравнения составного типа в трехмерной многосвязной области. Задача сведена к однозначно разрешимому интегральному уравнению. Решение задачи получено в форме динамических потенциалов, плотность в которых удовлетворяет этому уравнению. Тем самым доказана теорема существования. Кроме того, изучена единственность решения. Все результаты справедливы как для внутренних, так и для внешних областей с учетом соответствующих условий на бесконечности.

Библиографоия: 19 названий.

В динамике океана возникает эволюционное уравнение составного типа четвертого порядка, называемое уравнением гравитационно-гироскопических волн (см. (1.1)). Подобные уравнения имеют как эллиптические, так и гиперболические характеристики, а значит, обладают свойствами как эллиптических, так и гиперболических уравнений. Различные начально-краевые задачи для аналогичных уравнений исследовались в [1]-[12]. В частности, задачи для двумерного уравнения гравитационно-гироскопических волн в многосвязных областях изучались в [3], [7]-[12]. Для трехмерного уравнения гравитационно-гироскопических волн ранее изучались лишь начально-краевые задачи в односвязных областях [1], [2], [4]-[6]. Трехмерное уравнение в многосвязньг областях ранее не рассматривалось. Настоящая работа имеет целью восполнить этот пробел и изучить первую начально-краевую задачу в трехмерной многосвязной области (внутренней и внешней). Аналогичная задача в односвязной области сводилась в [1], [5], [6] к интегральньм уравнениям. Однако способ сведения к интегральным уравнениям, предложенный в этих работах для односвязной области, не проходит в случае многосвязной области, так как получающиеся интегральные уравнения могут не иметь решения, в то время как начально-краевая задача разрешима. Кроме того, способ сведения к интегральному уравнению, предлагаемьй в настоящей работе, отличается от ранее известного даже для внешней односвязной области. С помощью теории динамических потенциалов [1], [2] и метода граничных интегральных уравнений в работе доказана теорема существования решения для рассматриваемой задачи. Единственность решения также изучена. Следует отметить, что интегральное уравнение, полученное в настоящей работе, является однозначно разрешимым уравнением $Ф$ редгольма.

Работа выполнена при финансовой поддержке Российского фонда фундаментальных исследований, грант № 99-01-01063. 
1. Постановка задачи и теорема единственности. В декартовьх координа-

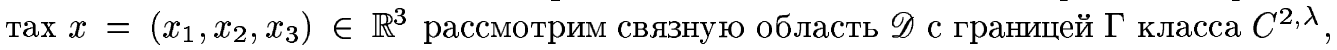
$\lambda \in(0,1]$. Будем считать, что $\Gamma$ состоит из простых непересекающихся замкнутых поверхностей $\Gamma_{0}, \Gamma_{1}, \ldots, \Gamma_{n} \in C^{2,0}$, так что $\Gamma_{0}$ охватьвает все остальные. Поверхность $\Gamma_{0}$ может отсутствовать, тогда область $\mathscr{D}$ будет неограниченной. Через $\boldsymbol{n}_{\boldsymbol{x}}$ обозначим внутреннюю по отношению к области $\mathscr{D}$ нормаль в точке $x \in \Gamma$. Рассмотрим поверхности, составляющие Г как двусторонние. Ту сторону поверхностей $Г$, которая видна, если глядеть навстречу концам нормальных векторов $\boldsymbol{n}_{x}, x \in \Gamma$, обозначим $\Gamma^{-}$, а противоположную сторону обозначим $\Gamma^{+}$.

Будем говорить, что функция $\mathscr{U}(t, x)$, определенная на множестве $[0, \infty) \times \overline{\mathscr{D}}$, принадлежит классу гладкости $G$, если

1) $\mathscr{U}, \mathscr{U}_{t} \in C^{0}([0, \infty) \times \mathscr{D}) ; \partial^{k} \partial^{p} \mathscr{U} / \partial t^{k} \partial x_{j}^{p} \in C^{0}((0, \infty) \times \mathscr{D}), k, p=0,1,2$, $j=1,2,3$;

2) при каждом $t \geqslant 0$ на гранище $\Gamma$ существует предельное значение функции $\mathscr{U}$ в смысле предельного перехода по нормали к границе.

$\Phi$ ункцию $\mathscr{U}(t, x)$, определенную в неограниченной области, назовем регулярной на бесконечности, если существуют такие функции $q_{1}(t), q_{2}(t) \in C^{0}[0, \infty)$, что при $|x| \rightarrow \infty$ справедливы неравенства

$$
\left|\frac{\partial^{j}}{\partial t^{j}} \mathscr{U}(t, x)\right| \leqslant q_{1}(t)|x|^{-1}, \quad\left|\frac{\partial^{j}}{\partial t^{j}} \mathscr{U}_{x_{k}}(t, x)\right| \leqslant q_{2}(t)|x|^{-2}, \quad j=0,1,2, \quad k=1,2,3 .
$$

Уравнение внутренних гравитационно-гироскопических волн в стратифицированной жидкости в случае трех пространственных переменных имеет вид [5], [13], [14]

$$
\frac{\partial^{2}}{\partial t^{2}} \Delta \mathscr{U}+\omega_{1}^{2} \Delta_{2} \mathscr{U}+\omega_{2}^{2} \mathscr{U}_{x_{3} x_{3}}=0, \quad \Delta=\frac{\partial^{2}}{\partial x_{1}^{2}}+\frac{\partial^{2}}{\partial x_{2}^{2}}+\frac{\partial^{2}}{\partial x_{3}^{2}}, \quad \Delta_{2}=\frac{\partial^{2}}{\partial x_{1}^{2}}+\frac{\partial^{2}}{\partial x_{2}^{2}}
$$

где $\omega_{1} \geqslant 0$ - частота Вяйсяля-Брента, $\omega_{2} \geqslant 0-$ удвоенная частота вращения вокруг оси $O x_{3}$.

Сформулируем первую начально-краевую задачу для уравнения (1.1) в области $\mathscr{D}$ при $t \geqslant 0$.

ЗАДАчА $\mathscr{K}$. Найти функцию $\mathscr{U}(t, x)$ из класса $G$, удовлетворяющую в классическом смысле уравнению $(1.1)$ в области $(0, \infty) \times \mathscr{D}$, начальным условиям

$$
\mathscr{U}(0, x)=\mathscr{U}_{t}(0, x)=0, \quad x \in \mathscr{D},
$$

и граничному условию на Г:

$$
\left.\mathscr{U}(t, x)\right|_{x \in \Gamma}=\left.f_{0}(t, x)\right|_{x \in \Gamma}, \quad t \geqslant 0 .
$$

Кроме того, если $\mathscr{D}$ - внешняя область, то функция $\mathscr{U}(t, x)$ должна быть регулярной на бесконечности.

Удовлетворение граничному условию понимается в смысле предельного перехода по нормали к границе $\Gamma$.

Наряду с классом $G$ рассмотрим класс гладкости $G_{1}$. 
Функция $\mathscr{U}(t, x) \in G_{1}$, если

1) $\mathscr{U}, \mathscr{U}_{t} \in C^{0}([0, \infty) \times \overline{\mathscr{D}}), \nabla \mathscr{U}, \nabla \mathscr{U}_{t} \in C^{0}([0, \infty) \times \mathscr{D})$,

$$
\frac{\partial^{k}}{\partial t^{k}} \frac{\partial^{p}}{\partial x_{j}^{p}} \mathscr{U} \in C^{0}((0, \infty) \times \mathscr{D}), \quad k, p=0,1,2, \quad j=1,2,3 ;
$$

$2)$ при каждом $t \geqslant 0$ на $Г$ существует равномерный по всем $x \in \Gamma$ предел выражения

$$
\begin{aligned}
\mathscr{N}_{t x} \mathscr{U}(t, \bar{x})= & \frac{\partial^{2}}{\partial t^{2}}\left(\boldsymbol{n}_{x} \nabla_{\bar{x}}\right) \mathscr{U}(t, \bar{x})+\omega_{1}^{2} \cos \left(\boldsymbol{n}_{x}, x_{1}\right) \mathcal{U}_{x_{1}}(t, \bar{x}) \\
& +\omega_{1}^{2} \cos \left(\boldsymbol{n}_{x}, x_{2}\right) \mathscr{U}_{x_{2}}(t, \bar{x})+\omega_{2}^{2} \cos \left(\boldsymbol{n}_{x}, x_{3}\right) \mathscr{U}_{x_{3}}(t, \bar{x})
\end{aligned}
$$

при $\bar{x}$, стремящемся к $x \in \Gamma$ по нормали $\boldsymbol{n}_{x}$, т. е. предел понимается в смысле предельного перехода по нормали к границе $\Gamma$. Через $\cos \left(\boldsymbol{n}_{x}, x_{j}\right)$ обозначен косинус угла между вектором нормали $\boldsymbol{n}_{x}$ в точке $x \in \Gamma$ и направлением оси $O x_{j}$, $j=1,2,3$.

Очевидно, что всякая функция из класса $G_{1}$ принадлежит классу $G$.

Всюду ниже под $\int_{\Gamma} \ldots d S$ понимается поверхностный интеграл первого рода.

Изучим вопрос о единственности решения задачи $\mathscr{K}$. Справедлива

Лемма 1. Пусть $\mathscr{U}(t, x)$ - классическое решение уравнения (1.1) в области $(0, \infty) \times \mathscr{D}$, принадлежсащее классу гладкости $G_{1}$. Кроме того, предположим, что функиия $\mathscr{U}(t, x)$ регулярна на бесконечности в случае внешней области $\mathscr{D}$. Тогда для любого $t \geqslant 0$ имеет место тождество

$$
\frac{1}{2} \frac{d}{d t}\left(\left\|\nabla \mathscr{U}_{t}\right\|^{2}+\omega_{1}^{2}\left\|\mathscr{U}_{x_{1}}\right\|^{2}+\omega_{1}^{2}\left\|\mathscr{U}_{x_{2}}\right\|^{2}+\omega_{2}^{2}\left\|\mathscr{U}_{x_{3}}\right\|^{2}\right)=\int_{\Gamma} \mathscr{U}_{t} \mathscr{N}_{t x} \mathscr{U} d s,
$$

где норма $\|\cdot\|$ берется в пространстве $L_{2}(\mathscr{D})$.

ДоКАЗАТЕЛЬСТвО леммы 1 проводится непосредственной проверкой. Для этого в области $\mathscr{D}$ построим эквидистантные поверхности [15] к границе Г. Если $\mathscr{D}$ - внешняя область, то помимо этого рассмотрим сферу достаточно большого радиуса $R$ с центром в начале координат. В области, ограниченной эквидистантными поверхностями (и сферой, если $\mathscr{D}$ - внешняя область), запишем энергетическое тождество, которое получается в результате умножения уравнения (1.1) на $\mathscr{U}_{t}$ и интегрирования по частям. Устремляя эквидистантные поверхности к границе Г и пользуясь свойствами функций класса $G_{1}$, докажем тождество из условия леммы в случае, когда $\mathscr{D}$ - внутренняя область. Если $\mathscr{D}$ - внешняя область, то в добавление к вышеизложенному устремим $R$ к бесконечности и используем регулярность $\mathscr{U}(t, x)$ на бесконечности. В результате снова получим требуемое тождество.

Непосредственно из леммы 1 вытекает

ТЕоремА 1. Если решение задачи $\mathscr{K}$ из класса гладкости $G_{1}$ существует, то оно единственно.

Разрешимость задачи $\mathscr{K}$ будет доказана при более слабых требованиях гладкости на ее решение, чем в теореме 1 , а именно, мы откажемся от требования принадлежности решения классу $G_{1}$.

Перейдем к построению классического решения задачи $\mathscr{K}$. 
2. Сведение задачи к интегральному уравнению. Введем некоторые классы функций. Положим

$$
C_{0}^{2}[0, \infty)=\left\{\varphi(t): \varphi(t) \in C^{2}[0, \infty), \varphi(0)=\varphi^{\prime}(0)=0\right\} .
$$

Через $C^{k}([0, \infty) ; \mathscr{H})$ обозначим класс $k$ раз непрерывно-дифференцируемых при $t \geqslant 0$ абстрактных функций $\varphi(t)$ со значениями в банаховом пространстве $\mathscr{H}$. Кроме того, введем следующий класс абстрактных функций:

$$
C_{0}^{2}([0, \infty) ; \mathscr{H})=\left\{\varphi(t): \varphi(t) \in C^{2}([0, \infty) ; \mathscr{H}), \varphi(0)=\varphi^{\prime}(0)=0\right\}
$$

Под обозначением $C^{0}([0, T] ; \mathscr{H})$, где $T>0$, будем понимать банахово пространство абстрактных функций, норма в котором определяется формулой

$$
\|\cdot\|_{C^{0}([0, T] ; \mathscr{H})}=\|\| \cdot\|\mathscr{H}\|_{C^{0}[0, T]} \cdot
$$

В дальнейшем из теории абстрактных функций нам понадобится следующий результат, схема доказательства которого приводится в [16, с. 28-29].

ЛЕмма 2. Классы функиий $C^{0}\left([0, \infty) ; C^{0}(\Gamma)\right)$ и $C^{0}([0, \infty) \times \Gamma)$ әквивалентны, т.е. всякая функция из первого класса принадлежит второму и наоборот.

Перейдем к построению решения задачи $\mathscr{K}$. Будем предполагать, что функция $f_{0}(t, x)$ из граничного условия (1.2) принадлежит классу $C_{0}^{2}\left([0, \infty) ; C^{0}(\Gamma)\right)$, тогда ее можно представить в виде

$$
f_{0}(t, x)=\int_{0}^{t} \int_{0}^{t_{1}} f\left(t_{2}, x\right) d t_{2} d t_{1}=\int_{0}^{t}(t-\tau) f(\tau, x) d \tau
$$

где

$$
f(t, x)=\frac{\partial^{2}}{\partial t^{2}} f_{0}(t, x) \in C^{0}\left([0, \infty) ; C^{0}(\Gamma)\right) .
$$

Через $J_{0}(\sigma)$ и $J_{1}(\sigma)$ будем обозначать функции Бесселя нулевого и первого порядка.

Рассмотрим функцию, введенную в [5] и названную динамическим потенииалом двойного слоя для уравнения (1.1):

$$
U[\bar{\mu}](t, x)=\int_{0}^{t} \int_{\Gamma} \bar{\mu}(\tau, y) \mathcal{N}_{\tau y} W(t-\tau, x-y) d S_{y} d \tau-\frac{1}{4 \pi} \int_{\Gamma} \bar{\mu}(t, y) \frac{\partial}{\partial \boldsymbol{n}_{y}} \frac{1}{|x-y|} d S_{y}
$$

где $x=\left(x_{1}, x_{2}, x_{3}\right), y=\left(y_{1}, y_{2}, y_{3}\right) \in \Gamma$, оператор $\mathscr{N}_{t x}$ определен в $(1.3)$,

$$
W(t, x)=-\frac{1}{4 \pi|x|_{0}} \int_{0}^{t|x|_{0} /|x|} J_{0}(\sigma) d \sigma=-\frac{t}{4 \pi|x|}+\frac{|x|_{0}}{4 \pi|x|^{2}}\left(t * J_{1}\left(t \frac{|x|_{0}}{|x|}\right)\right)
$$

- сингулярное решение для уравнения (1.1), $|x|=\left(x_{1}^{2}+x_{2}^{2}+x_{3}^{2}\right)^{1 / 2},|x|_{0}=\left(\omega_{2}^{2} x_{1}^{2}+\omega_{2}^{2} x_{2}^{2}+\right.$ $\left.\omega_{1}^{2} x_{3}^{2}\right)^{1 / 2}$. Знаком * обозначается операция свертки по времени, так что для любых двух функций времени $a(t), b(t)$ имеем

$$
a(t) * b(t)=\int_{0}^{t} a(t-\tau) b(\tau) d \tau=\int_{0}^{t} a(\tau) b(t-\tau) d \tau
$$


Относительно плотности $\bar{\mu}(t, x)$ будем предполагать, что она имеет вид

$$
\bar{\mu}(t, x)=\int_{0}^{t} \int_{0}^{t_{1}} \mu\left(t_{2}, x\right) d t_{2} d t_{1}=\int_{0}^{t}(t-\tau) \mu(\tau, x) d \tau
$$

где $\mu(t, x) \in C^{0}\left([0, \infty) ; C^{0}(\Gamma)\right)$, так что $\bar{\mu}(t, x) \in C_{0}^{2}\left([0, \infty) ; C^{0}(\Gamma)\right)$.

Как показано в [5], функция $W(t, x)$ при $x \neq 0$ удовлетворяет в классическом смысле уравнению (1.1), причем $W(0, x)=0, W_{t}(0, x)=-(4 \pi|x|)^{-1}$.

Для функции $\mathscr{N}_{\tau y} W(t-\tau, x-y)$ справедливо следующее представление [5]:

$$
\begin{aligned}
\mathscr{N}_{\tau y} W(t-\tau, x-y)= & \frac{\cos \left(x-y, \boldsymbol{n}_{y}\right)}{4 \pi|x-y|^{2}}\left[\frac{|x-y|_{0}}{|x-y|} J_{0}^{\prime}(\zeta)\right. \\
& +\frac{(\tau-t)\left(x_{3}-y_{3}\right)^{2}\left(|x-y|^{2}-\left(x_{3}-y_{3}\right)^{2}\right)}{|x-y|^{2}|x-y|_{0}^{2}}\left(\omega_{2}^{2}-\omega_{1}^{2}\right)^{2} J_{0}(\zeta) \\
& \left.+\frac{\omega_{2}^{2} \omega_{1}^{2}}{|x-y|_{0}^{3}}|x-y|^{3} \int_{0}^{\zeta} J_{0}(\sigma) d \sigma\right], \quad \zeta=\frac{(\tau-t)|x-y|_{0}}{|x-y|}
\end{aligned}
$$

Здесь $\left|\cos \left(x-y, \boldsymbol{n}_{y}\right)\right|$ - косинус угла между векторами $\vec{y} \vec{x}$ и $\boldsymbol{n}_{\boldsymbol{y}}$. Величина, стоящая в квадратных скобках, непрерывна при $x \neq y$ и равномерно ограничена по всем переменным на любом конечном промежутке времени. Поскольку $\left|\cos \left(x-y, \boldsymbol{n}_{y}\right)\right| \leqslant$ const $|x-y|^{\lambda_{1}}$ при $x, y \in \Gamma$ и $\Gamma \in C^{1, \lambda_{1}}$ (тем более, если $\Gamma \in C^{2, \lambda}$ как в нашем случае), то, как показано в [5, лемма 4.1.1], из результатов теории потенциала следует непрерьвность потенциала $U[\bar{\mu}](t, x)$ при $x \in \Gamma$.

Из свойств динамического потенциала двойного слоя, приведенных в [5], вытекает, что справедлива

ЛЕмма 3. Если $\mu(t, s) \in C^{0}\left([0, \infty) ; C^{0}(\Gamma)\right)$ u $\bar{\mu}(t, s)$ дается выражсением (2.2), то потенииал $U[\bar{\mu}](t, x)$ принадлежит классу $G$, удовлетворяет уравнению (1.1) в $(0, \infty) \times \mathscr{D}$ и начальным условиям задачи $\mathscr{K}$. Кроме того, если $\mathscr{D}$ - внешняя область, то потенииал $U[\bar{\mu}](t, x)$ регулярен на бесконечности.

Через $Y_{1}, \ldots, Y_{N}$ обозначим произвольные фиксированные точки, расположенные внутри поверхностей $\Gamma_{1}, \ldots, \Gamma_{N}$, и рассмотрим функцию

$$
\begin{aligned}
Q[\bar{\mu}](t, x)= & \sum_{k=1}^{N} \int_{0}^{t} \int_{\Gamma_{k}} \mu(\tau, y) d S_{y} W\left(t-\tau, x-Y_{k}\right) d \tau \\
= & \frac{1}{4 \pi} \sum_{k=1}^{N}\left\{\frac{-1}{\left|x-Y_{k}\right|} \int_{\Gamma_{k}} \bar{\mu}(t, y) d S_{y}\right. \\
& \left.+\frac{\left|x-Y_{k}\right|_{0}}{\left|x-Y_{k}\right|^{2}} \int_{0}^{t} \int_{\Gamma_{k}} \bar{\mu}(\tau, y) d S_{y} J_{1}\left(\frac{\left|x-Y_{k}\right|_{0}}{\left|x-Y_{k}\right|}(t-\tau)\right) d \tau\right\}
\end{aligned}
$$

где $W(t, x)$ определена вьше.

$\Phi$ ункция $Q[\bar{\mu}](t, x)$ является линейной комбинацией точечных источников для уравнения (1.1), расположенных в точках $Y_{k}, k=1, \ldots, N$. Если вьполнены условия леммы 3 , 
то $Q[\bar{\mu}](t, x)$ принадлежит классу $G$, удовлетворяет уравнению $(1.1)$ в $(0, \infty) \times \mathscr{D}$ и начальньм условиям задачи $\mathscr{K}$. Более того, $Q[\bar{\mu}](t, x)$ бесконечно дифференцируема по $x$ в $\mathbb{R}^{3} \backslash\left(\bigcup_{k=1}^{N} Y_{k}\right)$ и регулярна на бесконечности.

Решение задачи $\mathscr{K}$ ищем в виде

$$
\mathscr{U}[\bar{\mu}](t, x)=U[\bar{\mu}](t, x)+Q[\bar{\mu}](t, x)
$$

где $\bar{\mu}(t, x)$ определяется формулой $(2.2)$, для $U[\bar{\mu}](t, x)$ справедливо представление $(2.1)$, а $Q[\bar{\mu}](t, x)$ дается выражением (2.4).

Из указанных выше свойств функций, составляющих (2.5), вытекает

ЛЕммА 4. Если выполнены условия леммы 3, то функиия (2.5) принадлежит классу $G$, удовлетворяет уравнению $(1.1)$ в области $(0, \infty) \times \mathscr{D}$ и начальньм условиям задачи $\mathscr{K}$. Кроме того, если $\mathscr{D}$ - внешняя область, то функция (2.5) регулярна на бесконечности.

Таким образом, $\mathscr{U}[\bar{\mu}](t, x)$ удовлетворяет всем условиям задачи $\mathscr{K}$, кроме граничного условия (1.2). Пользуясь формулами для предельных значений динамического потенциала двойного слоя на границе области [5] и удовлетворяя граничному условию (1.2), приходим к интегральному уравнению относительно функции $\mu(t, x)$

$$
-\frac{1}{2} \mu(t, x)-(A \mu)(t, x)+(B \mu)(t, x)=f(t, x), \quad x \in \Gamma,
$$

где $f(t, x)=\left(f_{0}(t, x)\right)_{t t} \in C^{0}\left([0, \infty) ; C^{0}(\Gamma)\right), f_{0}(t, x) \in C_{0}^{2}\left([0, \infty) ; C^{0}(\Gamma)\right)$. Через $A$ и $B$ обозначены следуюшие операторы

$$
\begin{gathered}
(A \mu)(t, x)=\int_{\Gamma} \mu(t, y) \mathscr{A}(x, y) d S_{y}, \quad(B \mu)(t, x)=\left(B_{1} \mu\right)(t, x)-\left(B_{2} \mu\right)(t, x) \\
\left(B_{1} \mu\right)(t, x)=\int_{0}^{t} \int_{\Gamma} \mu(\tau, y) \mathscr{B}_{1}(t-\tau, x, y) d S_{y} d \tau, \quad\left(B_{2} \mu\right)(t, x)=\int_{0}^{t} \mu(\tau, x) \mathscr{B}_{2}(t-\tau) d \tau \\
\mathscr{B}_{1}(t-\tau, x, y)=\mathscr{N}_{\tau y} W(t-\tau, x-y)+\frac{1}{4 \pi} \sum_{k=1}^{N} \delta_{k}(y) \frac{\left|x-Y_{k}\right|_{0}}{\left|x-Y_{k}\right|^{2}} J_{1}\left((t-\tau) \frac{\left|x-Y_{k}\right|_{0}}{\left|x-Y_{k}\right|}\right) \\
\mathscr{B}_{2}(t)=\frac{1}{2} \omega_{1} \int_{0}^{\omega_{1} t} J_{1}(\sigma) \frac{d \sigma}{\sigma}, \quad \mathscr{A}(x, y)=\frac{1}{4 \pi} \frac{\partial}{\partial n_{y}} \frac{1}{|x-y|}+\frac{1}{4 \pi} \sum_{k=1}^{N} \delta_{k}(y) \frac{1}{\left|x-Y_{k}\right|} .
\end{gathered}
$$

Здесь $\mathscr{N}_{\tau y} W(t-\tau, x-y)-$ функция из $(2.3)$ и

$$
\delta_{k}(y)= \begin{cases}1, & y \in \Gamma_{k} \\ 0, & y \notin \Gamma_{k}\end{cases}
$$

Изучим некоторые свойства операторов $A$ и $B$. 
ЛЕмма 5.

1) $Я$ ра $\mathscr{A}(x, y), \mathscr{B}_{1}(t, x, y)$ непрерывны при $x \neq y$ и имеют слабую особенность nрu $x=y, \mathscr{B}_{2}(t) \in C^{0}[0, \infty)$.

2) Oператор $A$ действует из $C^{0}(\Gamma)$ в $C^{0}(\Gamma)$ и ограничен.

3) Onepaтор B действует из $C^{0}\left([0, \infty) ; C^{0}(\Gamma)\right)$ в $C^{0}\left([0, \infty) ; C^{0}(\Gamma)\right)$.

4) Если $h(t, x) \in C^{0}\left([0, \infty) ; C^{0}(\Gamma)\right)$, то при любом $t \geqslant 0$ справедливо неравенство

$\|(B h)(t, \cdot)\|_{C^{0}(\Gamma)} \leqslant c_{B}\left[\int_{0}^{t}\|h(\tau, \cdot)\|_{C^{0}(\Gamma)} d \tau+\int_{0}^{t} \int_{0}^{t_{1}} \| h(\tau, \cdot)_{C^{0}(\Gamma)} d \tau d t_{1}\right]$,

әде $c_{B}$ - некоторая константа.

ДокАЗАТЕЛЬСтво. Пункт 1) проверяется непосредственно. Ядро $\mathscr{A}(x, y)$ изучено в [15]. Свойства ядра $\mathscr{B}_{1}(t, x, y)$ вытекают из представления (2.3).

Пункт 2 ) следует из $[15, \S 17.4]]$ и п. 1$)$, поскольку ядро оператора $A$ - полярное.

Докажем п. 3). Пусть $h(t, x) \in C^{0}\left([0, \infty) ; C^{0}(\Gamma)\right)$. По лемме $2 h(t, x) \in C^{0}([0, \infty) \times \Gamma)$. Положим

$$
\begin{gathered}
\left(B_{1} h\right)(t, x)=(\hat{B} h)(t, x)+(\check{B} h)(t, x), \\
(\hat{B} h)(t, x)=\int_{0}^{t} \int_{\Gamma} h(\tau, y) \mathscr{N}_{\tau y} W(t-\tau, x-y) d S_{y} d \tau, \\
(\check{B} h)(t, x)=\int_{0}^{t} \int_{\Gamma} h(\tau, y)\left(\mathscr{B}_{1}(t-\tau, x, y)-\mathscr{N}_{\tau y} W(t-\tau, x-y)\right) d S_{y} d \tau,
\end{gathered}
$$

так что $B=\hat{B}+\check{B}+B_{2}$. Из результатов [5, лемма 4.1.1] следует, что оператор $\hat{B}$ действует из $C^{0}\left([0, \infty) ; C^{0}(\Gamma)\right)$ в $C^{0}\left([0, \infty) ; C^{0}(\Gamma)\right)$. Из явного выражения для ядер $\left(\mathscr{B}_{1}(t-\tau, x, y)-\mathscr{N}_{\tau} W(t-\tau, x-y)\right)$ и $\mathscr{B}_{2}(t)$ вытекает, что эти ядра непрерывны. Поэтому операторы $\ddot{B}$ и $B_{2}$ действуют из $C^{0}([0, \infty) \times \Gamma)$ в $C^{0}([0, \infty) \times \Gamma)$. С учетом леммы 2 операторы $\check{B}$ и $B_{2}$ действуют из $C^{0}\left([0, \infty) ; C^{0}(\Gamma)\right)$ в $C^{0}\left([0, \infty) ; C^{0}(\Gamma)\right)$. Тем самым, для оператора $B=\hat{B}+\check{B}+B_{2}$ справедлив п. 3) леммы.

Докажем п. 4). Пусть $\Gamma \in C^{1, \lambda_{1}} \subset C^{2, \lambda}, \lambda_{1} \in(0,1]$. При $x, y \in \Gamma$ справедливы оценки

$$
\begin{aligned}
\left|\mathscr{N}_{\tau y} W(t-\tau, x-y)\right| & \leqslant c_{1} \frac{\left|\cos \left(x-y, \boldsymbol{n}_{y}\right)\right|}{|x-y|^{2}}[1+(t-\tau)], \\
\mathscr{B}_{1}(t-\tau, x, y) & \leqslant c_{1} \frac{\left|\cos \left(x-y, \boldsymbol{n}_{y}\right)\right|}{|x-y|^{2}}[1+(t-\tau)]+c_{2},
\end{aligned}
$$

где $c_{1}, c_{2}-$ некоторые константы и первая оценка следует из (2.3).

Пусть $h(t, x) \in C^{0}\left([0, \infty) ; C^{0}(\Gamma)\right)$, тог да для оператора $B_{1}$ справедлива оценка

$$
\begin{aligned}
& \left|\left(B_{1} h\right)(t, x)\right| \leqslant \int_{0}^{t} \int_{\Gamma}|h(\tau, y)|\left\{c_{1} \frac{\left|\cos \left(x-y, \boldsymbol{n}_{y}\right)\right|}{|x-y|^{2}}[1+(t-\tau)]+c_{2}\right\} d S_{y} d \tau \\
& \quad \leqslant \int_{0}^{t}\|h(\tau, \cdot)\|_{C^{0}(\Gamma)}\left\{c_{1} \int_{\Gamma} \frac{\left|\cos \left(x-y, \boldsymbol{n}_{y}\right)\right|}{|x-y|^{2}} d S_{y}[1+(t-\tau)]+c_{2} \int_{\Gamma} 1 d S_{y}\right\} d \tau \\
& \quad \leqslant \int_{0}^{t}\|h(\tau, \cdot)\|_{C^{0}(\Gamma)}\left\{\widetilde{c}_{1}[1+(t-\tau)]+\widetilde{c}_{2}\right\} d \tau \\
& \quad=\widetilde{c}_{1} \int_{0}^{t} \int_{0}^{t_{1}}\|h(\tau, \cdot)\|_{C^{0}(\Gamma)} d \tau d t_{1}+\left(\widetilde{c}_{1}+\widetilde{c}_{2}\right) \int_{0}^{t}\|h(\tau, \cdot)\|_{C^{0}(\Gamma)} d \tau,
\end{aligned}
$$


где $\widetilde{c}_{1}, \widetilde{c}_{2}-$ некоторые постоянные и использована лемма 2 из $[15, \S 27.4]$ о том, что интеграл

$$
\int_{\Gamma} \frac{\left|\cos \left(x-y, \boldsymbol{n}_{y}\right)\right|}{|x-y|^{2}} d S_{y}
$$

равномерно ограничен константой для всех $x \in \Gamma$. Беря от обеих частей неравенства супремум по $x \in \Gamma$, получим

$$
\left\|\left(B_{1} h\right)(t, \cdot)\right\|_{C^{0}(\Gamma)} \leqslant\left(\widetilde{c}_{1}+\widetilde{c}_{2}\right)\left\{\int_{0}^{t} \int_{0}^{t_{1}}\|h(\tau, \cdot)\|_{C^{0}(\Gamma)} d \tau d t_{1}+\int_{0}^{t}\|h(\tau, \cdot)\|_{C^{0}(\Gamma)} d \tau\right\} .
$$

Аналогично,

$$
\left\|\left(B_{2} h\right)(t, \cdot)\right\|_{C^{0}(\Gamma)} \leqslant c_{0}\left\{\int_{0}^{t} \int_{0}^{t_{1}}\|h(\tau, \cdot)\|_{C^{0}(\Gamma)} d \tau d t_{1}\right\},
$$

где $c_{0}$ - некоторая постоянная и использована равномерная ограниченность функции $J_{1}(t) / t$ при $t \geqslant 0$. Складывая два последних неравенства, докажем оценку (2.7).

В следующем пункте продолжим изучение операторов, входящих в интегральное уравнение (2.6).

3. Свойства операторов, входящих в интегральное уравнение. Рассмотрим на контуре Г интегральное уравнение относительно функции $h(x) \in C^{0}(\Gamma)$

$$
-\frac{1}{2} h(x)-(A h)(x)=g(x), \quad x \in \Gamma,
$$

где $A$ - оператор из уравнения (2.6), а $g(x)$ принадлежит $C^{0}(\Gamma)$. По лемме 5 ядро оператора $A$ полярное, а значит, уравнение (3.1) представляет собой уравнение $\Phi$ редгольма второго рода. Докажем, что это уравнение однозначно разрешимо в $C^{0}(\Gamma)$. Для этого достаточно показать, что однородное уравнение (3.1) имеет только тривиальное решение. Доказательство проведем от противного. Пусть однородное уравнение имеет нетривиальное решение $h_{0}(x) \in C^{0}(\Gamma)$, для которого справедливо тождество

$$
-\frac{1}{2} h_{0}(x)-\left(A h_{0}\right)(x)=0, \quad x \in \Gamma .
$$

Запишем это тождество в виде

$$
-\frac{1}{2} h_{0}(x)=\int_{\Gamma} h_{0}(y) \mathscr{A}_{1}(x, y) d S_{y}+\int_{\Gamma} h_{0}(y) \mathscr{A}_{2}(x, y) d S_{y},
$$

где

$$
\mathscr{A}_{1}(x, y)=\frac{1}{4 \pi} \frac{\partial}{\partial n_{y}} \frac{1}{|x-y|}, \quad \mathscr{A}_{2}(x, y)=\frac{1}{4 \pi} \sum_{k=1}^{N} \delta_{k}(y) \frac{1}{\left|x-Y_{k}\right|} .
$$

Покажем, что $h_{0}(x) \in C^{1, \omega}(\Gamma)$ с $\omega \in(0,1)$. Действительно, второй интегральньй член в правой части (3.2) бесконечно дифференцируем по $x$, так как он не имеет особенности при $x=y$. Согласно [17, теорема 2.15] первьй интегральньй член в правой части (3.2) будет гёльдеровой функцией по $x$ на Г. Из тождества (3.2) для функции $h_{0}(x)$ вытекает, что $h_{0}(x)$ также будет гёльдеровой функцией по $x$ на $\Gamma$. Воспользовавшись $[17$, теорема 2.22], убеждаемся в том, что первый интегральный член в $(3.2)$ принадлежит $C^{1, \omega}(\Gamma)$ по $x$ для некоторого $\omega \in(0,1)$. Теперь из тождества $(3.2)$ для функции $h_{0}(x)$ получим $h_{0}(x) \in C^{1, \omega}(\Gamma), \omega \in(0,1)$. Тем самьм, доказана 
ЛЕмма 6. Всякое решение однородного уравнения (3.1) (сg三0) в $C^{0}(\Gamma)$ автоматически принадлежит $C^{1, \omega}(\Gamma)$, где $\omega \in(0,1)$.

Рассмотрим гармоническую в $\mathscr{D}$ функцию, построенную с использованием $h_{0}(x)$ и представляющую собой линейную комбинацию потенциала двойного слоя и точечных источников, расположенных внутри поверхностей $\Gamma_{k}$ в точках $Y_{k}$,

$$
v\left[h_{0}\right](x)=-\frac{1}{4 \pi} \int_{\Gamma} h_{0}(y) \frac{\partial}{\partial \boldsymbol{n}_{y}} \frac{1}{|x-y|} d S_{y}-\frac{1}{4 \pi} \sum_{k=1}^{N} \int_{\Gamma_{k}} h_{0}(y) d S_{y} \frac{1}{\left|x-Y_{k}\right|}
$$

Эта функция принадлежит $C^{0}(\overline{\mathscr{D}}) \cap C^{2}(\mathscr{D})$ и удовлетворяет всем условиям однородной задачи Дирихле

$$
\Delta v(x)=0, \quad x \in \mathscr{D},\left.\quad v\right|_{\Gamma}=0 .
$$

Кроме того, если $\mathscr{D}$ - внешняя область, то

$$
|v(x)|=O\left(|x|^{-1}\right) \quad \text { при }|x| \rightarrow \infty .
$$

Заметим, что при удовлетворении граничному условию $\left.v\right|_{\Gamma}=0$ получим тождество (3.2). В силу принципа максимума для гармонических функций решение этой однородной задачи Дирихле единственно. Следовательно,

$$
v\left[h_{0}\right](x) \equiv 0, \quad x \in \overline{\mathscr{D}}
$$

Через $\mathscr{D}_{j}$ обозначим внутреннюю односвязную область, ограниченную поверхностью $\Gamma_{j}, j=1, \ldots, N$. Пусть $\mathscr{J}$ - простая гладкая замкнутая поверхность, лежащая в области $\mathscr{D}$ и охватьвающая из всех поверхностей, составляющих $\Gamma$, только поверхность $\Gamma_{j}$. Полагая, что $\boldsymbol{n}_{\boldsymbol{x}}$ - непрерьвное поле нормалей на поверхности $\mathscr{J}$, вычислим

$$
\begin{aligned}
\int_{\mathscr{J}} \frac{\partial v\left[h_{0}\right](x)}{\partial \boldsymbol{n}_{x}} d S_{x}=0 & =-\int_{\Gamma_{j}} h_{0}(y) \frac{\partial}{\partial \boldsymbol{n}_{y}}\left(\frac{1}{4 \pi} \int_{\mathscr{J}} \frac{\partial}{\partial \boldsymbol{n}_{x}} \frac{1}{|x-y|} d S_{x}\right) d S_{y} \\
& -\int_{\Gamma_{j}} h_{0}(y) d S_{y} \frac{1}{4 \pi} \int_{\mathscr{J}} \frac{\partial}{\partial \boldsymbol{n}_{x}} \frac{1}{\left|x-Y_{j}\right|} d S_{x} .
\end{aligned}
$$

Здесь учтено следующее свойство гармонических функций: если функция $V(x)$ гармоническая внутри поверхности $\mathscr{J}$ и $\nabla V(x)$ непрерывно продолжается на $\mathscr{J}$ изнутри, то

$$
\int_{\mathscr{J}} \frac{\partial V}{\partial \boldsymbol{n}_{x}} d S_{x}=0
$$

Примем во внимание, что по формуле Остроградского-Гаусса

$$
\frac{1}{4 \pi} \int_{\mathscr{J}} \frac{\partial}{\partial \boldsymbol{n}_{x}} \frac{1}{|x-y|} d S_{x}=\frac{1}{4 \pi} \int_{\mathscr{J}} \frac{\partial}{\partial \boldsymbol{n}_{x}} \frac{1}{\left|x-Y_{j}\right|} d S_{x}= \pm 1
$$


где знак зависит от выбора направления поля нормалей $\boldsymbol{n}_{\boldsymbol{x}}$ на поверхности $\mathscr{J}$. Следовательно,

$$
\int_{\mathscr{J}} \frac{\partial v\left[h_{0}\right](x)}{\partial \boldsymbol{n}_{x}} d S_{x}=\mp \int_{\Gamma_{j}} h_{0}(y) d S_{y}=0 .
$$

В результате получаем тождество

$$
\int_{\Gamma_{j}} h_{0}(y) d S_{y}=0
$$

которое гарантирует, что функция $v\left[h_{0}\right](x)$ не содержит внутри поверхности $\Gamma_{j}$ точечного источника. Поэтому $v\left[h_{0}\right](x)$ - гармоническая в $\mathscr{D}_{j}$ функция. По лемме $6 h_{0}(x) \in$ $C^{1, \omega}(\Gamma)$. Как показано в $\left[17\right.$, теорема 2.23], при такой плотности $h_{0}(x)$ первые производные потенциала двойного слоя в $v\left[h_{0}\right](x)$ непрерывно продолжимы на $\Gamma_{j}$ изнутри и извне. Более того,

$$
\lim _{\substack{x^{1} \rightarrow x \in \Gamma_{j} \\ x^{1} \in \mathscr{D}}} \frac{\partial v\left[h_{0}\right]\left(x^{1}\right)}{\partial \boldsymbol{n}_{\boldsymbol{x}}}=\lim _{\substack{x^{1} \rightarrow x \in \Gamma_{j} \\ x^{1} \in \mathscr{D}_{j}}} \frac{\partial v\left[h_{0}\right]\left(x^{1}\right)}{\partial \boldsymbol{n}_{x}}=0,
$$

где учтено (3.3). Очевидно, функция $v\left[h_{0}\right](x)$ удовлетворяет следующей задаче Неймана в области $\mathscr{D}_{j}$ :

$$
\Delta v(x)=0, \quad x \in \mathscr{D}_{j} ;\left.\quad \frac{\partial v}{\partial \boldsymbol{n}_{x}}\right|_{\Gamma_{j}}=0 .
$$

Известно [15], что решением такой задачи является константа, т.е.

$$
v\left[h_{0}\right](x) \equiv \text { const }=c, \quad x \in \mathscr{D}_{j}
$$

Применяя теорему о скачке потенциала двойного слоя и пользуясь $(3.3)$, получим

$$
\lim _{\substack{x^{1} \rightarrow x \in \Gamma_{j} \\ x^{1} \in \mathscr{D}}} v\left[h_{0}\right]\left(x^{1}\right)-\lim _{\substack{x^{1} \rightarrow x \in \Gamma_{j} \\ x \in \mathscr{D}_{j}}} v\left[h_{0}\right]\left(x^{1}\right)=-h_{0}(x)=-c,
$$

так что $h_{0}(x)=c$ на $\Gamma_{j}$. Из $(3.4)$ вытекает, что $c=0$. Поэтому $h_{0}(x) \equiv 0$ на $\Gamma_{j}$. Напомним, что $\Gamma_{j}, j=1, \ldots, N,-$ произвольная замкнутая поверхность, принадлежащая $\Gamma$. Следовательно, $h_{0}(x) \equiv 0$ на $\Gamma_{1}, \ldots, \Gamma_{N}$. Таким образом, $h_{0}(x) \equiv 0$ на $\Gamma$, если $\mathscr{D}$-внешняя область, и $h_{0}(x) \equiv 0$ на $\Gamma \backslash \Gamma_{0}$, если $\mathscr{D}$ - внутренняя область. Осталось выяснить, чему равно $h_{0}(x)$ на $\Gamma_{0}$ в случае, когда $\mathscr{D}-$ внутренняя область.

Пусть $\mathscr{D}$ - внутренняя область; тогда $h_{0}(x) \equiv 0$ на $\Gamma \backslash \Gamma_{0}$ и функция $v\left[h_{0}\right](x)$ принимает вид потенциала двойного слоя

$$
v\left[h_{0}\right](x)=-\frac{1}{4 \pi} \int_{\Gamma_{0}} h_{0}(y) \frac{\partial}{\partial n_{y}} \frac{1}{|x-y|} d S_{y} .
$$

Пусть $\mathscr{D}_{0}$ - внешняя односвязная область, ограниченная поверхностью $\Gamma_{0}$. Потенциал $v\left[h_{0}\right](x)$ гармонический в $\mathscr{D}_{0}$ и $v(x)=O\left(|x|^{-1}\right), \nabla v(x)=O\left(|x|^{-2}\right)$ при $|x| \rightarrow \infty$. Учитывая гладкость $h_{0}(x)$ на $Г$, гарантированную леммой 6 , и применяя [14, теорема 2.23], получим

$$
\lim _{\substack{x^{1} \rightarrow x \in \Gamma_{0} \\ x^{1} \in \mathscr{D}}} \frac{\partial v\left[h_{0}\right]\left(x^{1}\right)}{\partial \boldsymbol{n}_{x}}=\lim _{\substack{x^{1} \rightarrow x \in \Gamma_{0} \\ x^{1} \in \mathscr{D}_{0}}} \frac{\partial v\left[h_{0}\right]\left(x^{1}\right)}{\partial \boldsymbol{n}_{x}}=0,
$$


где использовано (3.3). Следовательно, функция $v\left[h_{0}\right](x)$ удовлетворяет следующей задаче Неймана в $\mathscr{D}_{0}$ :

$$
\begin{gathered}
\Delta v(x)=0, \quad x \in \mathscr{D}_{0},\left.\quad \frac{\partial v}{\partial \boldsymbol{n}_{x}}\right|_{\Gamma_{0}}=0, \\
v(x)=O\left(|x|^{-1}\right), \quad \nabla v(x)=O\left(|x|^{-2}\right), \quad|x| \rightarrow \infty .
\end{gathered}
$$

Известно [15], что эта задача имеет только тривиальное решение: $v\left[h_{0}\right](x) \equiv 0$ в $\mathscr{D}_{0}$. Пользуясь теоремой о скачке потенциала двойного слоя [15] и пользуясь (3.3), получим

$$
\lim _{\substack{x^{1} \rightarrow x \in \Gamma_{0} \\ x^{1} \in \mathscr{D}}} v\left[h_{0}\right]\left(x^{1}\right)-\lim _{\substack{x^{1} \rightarrow x \in \Gamma_{0} \\ x \in \mathscr{D}_{0}}} v\left[h_{0}\right]\left(x^{1}\right)=-h_{0}(x)=0,
$$

так что $h_{0}(x) \equiv 0$ на $\Gamma_{0}$, если $\Gamma_{0} \subset \Gamma$. С учетом предыдущих результатов $h_{0}(x) \equiv 0$ на $\Gamma$ как в случае внешней, так и в случае внутренней области $\mathscr{D}$.

Тем самым мы приходим к противоречию с предположением о том, что $h_{0}(x)$ - нетривиальное решение однородного уравнения (3.1) (с $g \equiv 0)$. Таким образом, однородное уравнение (3.1) имеет только тривиальное решение. Ввиду фредгольмовости уравнения (3.1) справедлива

ЛЕмма 7. Уравнение (3.1) однозначно разрешимо в $C^{0}(\Gamma)$ при любой правой части $g(x) \in C^{0}(\Gamma)$.

Оператор, обратньй к $(-1 / 2-A)$, обозначим $(-1 / 2-A)^{-1}$, тогда решение уравнения (3.1) запишем в виде

$$
h(x)=\left(\left(-\frac{1}{2}-A\right)^{-1} g\right)(x) .
$$

Пусть $g(t, x) \in C^{0}\left([0, \infty) ; C^{0}(\Gamma)\right)$. Рассмотрим аналог уравнения (3.1) относительно функции $h(t, x) \in C^{0}\left([0, \infty) ; C^{0}(\Gamma)\right)$

$$
-\frac{1}{2} h(t, x)-(A h)(t, x)=g(t, x) \text {. }
$$

Для каждого фиксированного $t$ оператор $(-1 / 2-A)$ обратим,

$$
h(t, x)=\left(\left(-\frac{1}{2}-A\right)^{-1} g\right)(t, x)
$$

и при каждом $t$ вьполняется условие $h(t, x) \in C^{0}(\Gamma)$.

Поскольку оператор $(-1 / 2-A)$ линейньй и ограниченньй в $C^{0}(\Gamma)$ (см. п. 2 леммы 5$)$, то по теореме Банаха [18, с. 59] линейный оператор $(-1 / 2-A)^{-1}$ также ограничен в $C^{0}(\Gamma)$. Поэтому при каждом $t \geqslant 0$ имеет место оценка

$$
\|h(t, \cdot)\|_{C^{0}(\Gamma)} \leqslant c_{A}\|g(t, \cdot)\|_{C^{0}(\Gamma)},
$$

где $c_{A}$ - константа. Из ограниченности оператора $(-1 / 2-A)^{-1}$ следует его непрерывность. Это значит, что если

$$
\left\|g(t, \cdot)-g\left(t_{0}, \cdot\right)\right\|_{C^{0}(\Gamma)} \rightarrow 0, \quad t \rightarrow t_{0}
$$

то для функций $h(t, x), h\left(t_{0}, x\right)$, найденных по формуле $(3.6)$, справедливо соотношение

$$
\left\|h(t, \cdot)-h\left(t_{0}, \cdot\right)\right\|_{C^{0}(\Gamma)} \rightarrow 0, \quad t \rightarrow t_{0}
$$

Отсюда следует, что $h(t, x) \in C^{0}\left([0, \infty) ; C^{0}(\Gamma)\right)$. Тем самым, справедлива 
Лемма 8. Ecли $g(t, x) \in C^{0}\left([0, \infty) ; C^{0}(\Gamma)\right)$, то уравнение $(3.5)$ имеет единственное решение $h(t, x)$ из класса $C^{0}\left([0, \infty) ; C^{0}(\Gamma)\right)$, которое удовлетворяет оценке (3.7).

4. Решение интегрального уравнения и теорема существования. Решение интегрального уравнения (2.6) будем искать в виде ряда [6]

$$
\mu(t, x)=\sum_{m=0}^{\infty} \mu_{m}(t, x)
$$

где функции $\mu_{m}(t, x) \in C^{0}\left([0, \infty) ; C^{0}(\Gamma)\right)$ определяются последовательно из равенств, получающихся после подстановки (4.1) в (2.6):

$$
\begin{gathered}
-\frac{1}{2} \mu_{0}(t, x)-\left(A \mu_{0}\right)(t, x)=f(t, x), \\
-\frac{1}{2} \mu_{m}(t, x)-\left(A \mu_{m}\right)(t, x)=-\left(B \mu_{m-1}\right)(t, x), \quad m=1,2, \ldots
\end{gathered}
$$

Поскольку оператор $A$ не зависит от $t$, эта переменная в уравнениях (4.2), (4.3) играет роль параметра.

Так как функция $f(t, x) \in C^{0}\left([0, \infty) ; C^{0}(\Gamma)\right)$, то согласно лемме 8 уравнение $(4.2)$ имеет единственное решение $\mu_{0}(t, x) \in C^{0}\left([0, \infty) ; C^{0}(\Gamma)\right)$, для которого справедливо неравенство

$$
\left\|\mu_{0}(t, \cdot)\right\|_{C^{0}(\Gamma)} \leqslant c_{A}\|f(t, \cdot)\|_{C^{0}(\Gamma)} \leqslant c_{A}\|f\|_{C^{0}\left([0, t] ; C^{0}(\Gamma)\right)} \cdot
$$

Норма в банаховом пространстве $C^{0}\left([0, T] ; C^{0}(\Gamma)\right)$ определяется соотношением

$$
\|\cdot\|_{C^{0}\left([0, T] ; C^{0}(\Gamma)\right)}=\|\| \cdot\left\|_{C^{0}(\Gamma)}\right\|_{C^{0}[0, T]} .
$$

Подставляя найденное $\mu_{0}(t, x)$ в правую часть уравнения $(4.3)$, получим уравнение относительно $\mu_{1}(t, x)$ :

$$
-\frac{1}{2} \mu_{1}(t, x)-\left(A \mu_{1}\right)(t, x)=-\left(B \mu_{0}\right)(t, x) .
$$

В силу п. 3) леммы 5 выполняется условие $\left(B \mu_{0}\right)(t, x) \in C^{0}\left([0, \infty) ; C^{0}(\Gamma)\right)$. Согласно лемме 8 уравнение (4.5) имеет единственное решение

$$
\mu_{1}(t, x) \in C^{0}\left([0, \infty) ; C^{0}(\Gamma)\right)
$$

Кроме того, по леммам 5,8 функция $\mu_{1}(t, x)$ удовлетворяет оценке

$$
\begin{aligned}
\left\|\mu_{1}(t, \cdot)\right\|_{C^{0}(\Gamma)} & \leqslant c_{A}\left\|\left(B \mu_{0}\right)(t, \cdot)\right\|_{C^{0}(\Gamma)} \\
& \leqslant c_{A} c_{B}\left[\int_{0}^{t}\left\|\mu_{0}(\tau, \cdot)\right\|_{C^{0}(\Gamma)} d \tau+\int_{0}^{t} \int_{0}^{t_{1}}\left\|\mu_{0}(\tau, \cdot)\right\|_{C^{0}(\Gamma)} d \tau d t_{1}\right] .
\end{aligned}
$$


Далее рассуждения проводятся по индукции. Пусть $\mu_{m-1}(t, x) \in C^{0}\left([0, \infty) ; C^{0}(\Gamma)\right)$, тог да $\mu_{m}(t, x)$ должно удовлетворять уравнению (4.3). По лемме 5

$$
\left(B \mu_{m-1}\right)(t, x) \in C^{0}\left([0, \infty) ; C^{0}(\Gamma)\right) .
$$

По лемме 8 уравнение (4.3) имеет единственное решение $\mu_{m}(t, x) \in C^{0}\left([0, \infty) ; C^{0}(\Gamma)\right)$. По леммам 5,8 это решение удовлетворяет оценке

$$
\begin{aligned}
\left\|\mu_{m}(t, \cdot)\right\|_{C^{0}(\Gamma) \leqslant} & c_{A} c_{B}\left[\int_{0}^{t}\left\|\mu_{m-1}(\tau, \cdot)\right\|_{C^{0}(\Gamma)} d \tau+\int_{0}^{t} \int_{0}^{t_{1}}\left\|\mu_{m-1}(\tau, \cdot)\right\|_{C^{0}(\Gamma)} d \tau d t_{1}\right] \\
\leqslant & c_{A}^{2} c_{B}^{2}\left[\int_{0}^{t} \int_{0}^{t_{1}}\left\|\mu_{m-2}(\tau, \cdot)\right\|_{C^{0}(\Gamma)} d \tau d t_{1}\right. \\
& \left.+\int_{0}^{t} \int_{0}^{t_{1}} \int_{0}^{t_{2}} \int_{0}^{t_{3}}\left\|\mu_{m-2}(\tau, \cdot)\right\|_{C^{0}(\Gamma)} d \tau d t_{3} d t_{2} d t_{1}\right] \leqslant \ldots \\
\leqslant & c_{A}^{m} c_{B}^{m}\left[\int_{0}^{t} \int_{0}^{t_{1}} \cdots \int_{0}^{t_{m-1}}\left\|\mu_{0}(\tau, \cdot)\right\|_{C^{0}(\Gamma)} d \tau d t_{m-1} \ldots d t_{1}\right. \\
& \left.+\int_{0}^{t} \int_{0}^{t_{1}} \ldots \int_{0}^{t_{2 m-1}}\left\|\mu_{0}(\tau, \cdot)\right\|_{C^{0}(\Gamma)} d \tau d t_{2 m-1} \ldots d t_{1}\right] \\
\leqslant & c_{A}^{m+1} c_{B}^{m}\|f\|_{C^{0}\left([0, t] ; C^{0}(\Gamma)\right)}\left[\int_{0}^{t} \int_{0}^{t_{1}} \ldots \int_{0}^{t_{m-1}} d \tau d t_{m-1} \ldots d t_{1}\right. \\
& \left.+\int_{0}^{t} \int_{0}^{t_{1}} \ldots \int_{0}^{t_{2 m-1}} d \tau d t_{2 m-1} \ldots d t_{1}\right] \\
= & c_{A}\|f\|_{C^{0}\left([0, t] ; C^{0}(\Gamma)\right)}\left(\frac{\left(c_{B} c_{A} t\right)^{m}}{m !}+\frac{\left(\sqrt{c_{B} c_{A}} t\right)^{2 m}}{(2 m) !}\right)
\end{aligned}
$$

где использовано неравенство (4.4).

Таким образом, все функции $\mu_{m}(t, x)$, образующие ряд (4.1), находятся последовательно. В силу леммы 2 каждая из функций $\mu_{m}(t, x)$ принадлежит $C^{0}([0, \infty) \times \Gamma)$. Кроме того, каждая из них удовлетворяет неравенству (4.6).

Покажем, что ряд (4.1) сходится абсолютно и равномерно в $[0, T] \times \Gamma$ при любом $T>0$. Для этого воспользуемся теоремой Вейерштрасса [19] и замажорируем члены ряда (4.1) членами сходящегося числового ряда. Применяя неравенство (4.6), получим, что для любых $(t, x) \in[0, T] \times \Gamma$ справедливо неравенство

$$
\begin{aligned}
\left|\mu_{m}(t, x)\right| & \leqslant\left\|\mu_{m}(t, \cdot)\right\|_{C^{0}(\Gamma)} \leqslant \sup _{t \in[0, T]}\left\|\mu_{m}(t, \cdot)\right\|_{C^{0}(\Gamma)}=\left\|\mu_{m}\right\|_{C^{0}\left([0, T] ; C^{0}(\Gamma)\right)} \\
& \leqslant c_{A}\left[\frac{\left(c_{A} c_{B} T\right)^{m}}{m !}+\frac{\left(\sqrt{c_{A} c_{B}} T\right)^{2 m}}{(2 m) !}\right]\|f\|_{C^{0}\left([0, T] ; C^{0}(\Gamma)\right)} .
\end{aligned}
$$

Тем самым каждый член ряда (4.1) мажорируется на множестве $[0, T] \times \Gamma$ членом числового ряда

$$
c_{A}\|f\|_{C^{0}\left([0, T] ; C^{0}(\Gamma)\right)}\left[\frac{\left(c_{A} c_{B} T\right)^{m}}{m !}+\frac{\left(\sqrt{c_{A} c_{B}} T\right)^{2 m}}{(2 m) !}\right],
$$

который сходится и его сумма равна

$$
c_{A}\|f\|_{C^{0}\left([0, T] ; C^{0}(\Gamma)\right)}\left[\exp \left(c_{A} c_{B} T\right)+\operatorname{ch}\left(\sqrt{c_{A} c_{B}} T\right)\right] .
$$


Следовательно, по теореме Вейерштрасса ряд (4.1) сходится абсолютно и равномерно на множестве $[0, T] \times \Gamma$ при любом $T>0$. Отсюда с учетом непрерывности членов ряда вытекает возможность почленного интегрирования ряда при подстановке его в уравнение (2.6). При этом ряды, полученные в результате, также будут сходиться абсолютно и равномерно $[19]$ в $[0, T] \times \Gamma$ при любом $T>0$, а значит, их члены можно суммировать в произвольном порядке. В силу справедливости цепочки равенств (4.2), (4.3) уравнение (2.6) обращается в тождество. Тем самым функция $\mu(t, x)$, определяемая как сумма ряда (4.1), действительно удовлетворяет уравнению (2.6), потому что все формальные операции по подстановке (4.1) в (2.6) обоснованы.

По теореме о непрерывности суммы ряда [19] из равномерной сходимости ряда (4.1) в $[0, T] \times \Gamma$ при любом $T>0$ и непрерьвности его членов в $[0, \infty) \times \Gamma$ следует, что $\mu(t, x) \in C^{0}([0, \infty) \times \Gamma)$. В свою очередь, по лемме 2 вьполняется условие $\mu(t, x) \in$ $C^{0}\left([0, \infty) ; C^{0}(\Gamma)\right)$. В результате искомое решение уравнения $(2.6)$ построено.

ТЕорема 2. При любой функиии $f(t, x) \in C^{0}\left([0, \infty) ; C^{0}(\Gamma)\right)$ уравнение $(2.6)$ имеет решение $\mu(t, x) \in C^{0}\left([0, \infty) ; C^{0}(\Gamma)\right)$.

Заметим, что всякой функции $f_{0}(t, x) \in C_{0}^{2}\left([0, \infty) ; C^{0}(\Gamma)\right)$ из граничного условия $(1.2)$ задачи $\mathscr{K}$ отвечает функция $f(t, x)=\left[f_{0}(t, x)\right]_{t t} \in C^{0}\left([0, \infty) ; C^{0}(\Gamma)\right)$, стоящая в правой части интегрального уравнения (2.6).

Теорема 2 гарантирует существование решения $\mu(t, x)$ интегрального уравнения (2.6) из требуемого класса гладкости. Следовательно, потенциал $(2.5)$, построенньй на этой функции $\mu(t, x)$, удовлетворяет граничному условию задачи $\mathscr{K}$. Кроме того, по лемме 4 построенньй таким образом потенциал (2.5) принадлежит классу гладкости $G$ и удовлетворяет всем остальным условиям задачи $\mathscr{K}$. Справедлива

ТЕорема 3. При любой функиии $f_{0}(t, x) \in C_{0}^{2}\left([0, \infty) ; C^{0}(\Gamma)\right)$ классическое решение задачи $\mathscr{K}$ существует и дается формулой $(2.5)$, где плотность $\mu(t, x) \in$ $C^{0}\left([0, \infty) ; C^{0}(\Gamma)\right)$ является решением интегрального уравнения $(2.6)$, которое всегда разрешимо.

Можно показать, что если $f_{0}(t, x) \in C_{0}^{2}\left([0, \infty) ; C^{1, \lambda}(\Gamma)\right), \lambda \in(0,1]$, то решение задачи $\mathscr{K}$, построенное в теореме 3, принадлежит классу гладкости $G_{1}$. В этом случае по теореме 1 решение задачи $\mathscr{K}$ единственно.

5. О фредгольмовости интегрального уравнения. В предыдущем пункте была установлена разрешимость интегрального уравнения (2.6), однако остался открытым вопрос о числе его решений. Покажем, что уравнение (2.6) однозначно разрешимо, так как это, в частности, очень важно при численной реализации предлагаемого метода.

Рассмотрим уравнение $(2.6)$ в банаховом пространстве $C^{0}\left([0, T] ; C^{0}(\Gamma)\right)$ при произвольном $T>0$.

Пусть $\mu(t, x) \in C^{0}\left([0, T] ; C^{0}(\Gamma)\right)$, тогда функция $(A \mu)(t, x)$ непрерывна при каждом $t$ по $x$. Кроме того, принимая во внимание п. 1 леммы 5, имеем

$$
\begin{gathered}
\left\|(A \mu)(t, \cdot)-(A \mu)\left(t_{0}, \cdot\right)\right\|_{C^{0}(\Gamma)} \leqslant \text { const }\left\|\mu(t, \cdot)-\mu\left(t_{0}, \cdot\right)\right\|_{C^{0}(\Gamma)}, \\
\|(A \mu)(\cdot, \cdot)\|_{C^{0}\left([0, T] ; C^{0}(\Gamma)\right)} \leqslant \text { const }\|\mu(\cdot, \cdot)\|_{C^{0}\left([0, T] ; C^{0}(\Gamma)\right)} .
\end{gathered}
$$

С учетом гладкости функции $\mu(t, x)$ отсюда следует, что оператор $A$ отображает пространство $C^{0}\left([0, T] ; C^{0}(\Gamma)\right)$ в себя и при этом ограничен. Из леммы 8 вытекает обратимость оператора $(-1 / 2-A)$ в $C^{0}\left([0, T] ; C^{0}(\Gamma)\right)$, а из теоремы Банаха [18, с. 59]ограниченность оператора $(-1 / 2-A)^{-1}$ в этом пространстве. 
Воспользовавшись аналогом теоремы Арцела для абстрактных функций [16, с. 32], несложно показать, что оператор $B$ из (2.6) компактен как оператор, отображающий пространство $C^{0}\left([0, T] ; C^{0}(\Gamma)\right)$ в себя (доказательство дано в п. 6). С учетом обратимости оператора $(-1 / 2-A)$ заметим, что уравнение (2.6) эквивалентно следующему уравнению

$$
\mu(t, x)+\left(\left(-\frac{1}{2}-A\right)^{-1} B \mu\right)(t, x)=\left(\left(-\frac{1}{2}-A\right)^{-1} f\right)(t, x),
$$

г де $\mu(t, x),\left((-1 / 2-A)^{-1} f\right)(t, x) \in C^{0}\left([0, T] ; C^{0}(\Gamma)\right)$.

В силу отмеченных вьше ограниченности оператора $(-1 / 2-A)^{-1}$ и компактности оператора $B$ в $C^{0}\left([0, T] ; C^{0}(\Gamma)\right)$ оператор $(-1 / 2-A)^{-1} B$ будет компактным в этом пространстве [18], а уравнение (5.1) является в нем уравнением Фредгольма второго рода. Очевидно, что уравнения (2.6) и (5.1) имеют одни и те же решения.

Вследствие обратимости оператора $(-1 / 2-A)^{-1}$ областью его значений будет все пространство $C^{0}\left([0, T] ; C^{0}(\Gamma)\right)$. Таким образом, функция $\left((-1 / 2-A)^{-1} f\right)(t, x)$, стоящая в правой части (5.1), может быть произвольной функцией из пространства $C^{0}([0, T]$; $\left.C^{0}(\Gamma)\right)$. И для этой произвольной функции уравнение (5.1) разрешимо, так как по теореме 2 разрешимо эквивалентное ему уравнение (2.6). Из альтернативы Фредгольма вытекает, что безусловно разрешимое уравнение Фредгольма является однозначно разрешимым. Следовательно, (5.1) - однозначно разрешимое уравнение, а значит, и эквивалентное ему уравнение (2.6) тоже однозначно разрешимо. Вследствие произвольности $T>0$ приходим к следующей теореме.

ТЕОРемА 4. Уравнение (2.6) әквивалентно уравнению Фредгольма (5.1) и однозначно разрешимо в $C^{0}\left([0, \infty) ; C^{0}(\Gamma)\right)$.

\section{6. О компактности оператора $B$. В этом пункте будет доказана}

Лемма 9. При любом $T>0$ оператор $B$ компактен как оператор, действующий в $C^{0}\left([0, T] ; C^{0}(\Gamma)\right)$.

Для доказательства леммы достаточно доказать компактность операторов $B_{1}$ и $B_{2}$ в банаховом пространстве $C^{0}\left([0, T] ; C^{0}(\Gamma)\right)$, тогда и оператор $B$ будет компактньм.

Пусть $M$ - ограниченное множество абстрактных функций $h(t, x)$ в банаховом пространстве $C^{0}\left([0, T] ; C^{0}(\Gamma)\right)$, т. е. существует константа $c_{M}$ такая, что

$$
\|h(\cdot, \cdot)\|_{C^{0}\left([0, T] ; C^{0}(\Gamma)\right)}<c_{M}, \quad h \in M .
$$

Операторы $B_{1}$ и $B_{2}$ будут компактными, если множества $B_{1} M$ и $B_{2} M$ компактны. Для выяснения компактности этих множеств воспользуемся следующим аналогом теоремы Арцела для абстрактных функций [16, с. 32].

УТВЕРЖДЕНИЕ. Для того чтобы множество $\mathscr{L} \in C^{0}\left([0, T] ; C^{0}(\Gamma)\right)$ было компактным, необходимо и достаточно выполнения двух условий:

1) функиии мнохества $\mathscr{L}$ равномерно ограничены, т. е. существует такая константа с, что

$$
\|g(\cdot, \cdot)\|_{C^{0}\left([0, T] ; C^{0}(\Gamma)\right)}<c, \quad g(t, x) \in \mathscr{L}
$$


2) функиии множсества $\mathscr{L}$ равностепенно непрерывны, т.е. для любого $\varepsilon>0$ найдется такое $\delta>0$, что если $\left|t_{2}-t_{1}\right|<\delta ; t_{1}, t_{2} \in[0, T]$, то сразу для всех $g(t, x) \in \mathscr{L}$ справедливо неравенство

$$
\left\|g\left(t_{2}, \cdot\right)-g\left(t_{1}, \cdot\right)\right\|_{C^{0}(\Gamma)}<\varepsilon .
$$

Равномерную ограниченность множеств $B_{1} M$ и $B_{2} M$ в $C^{0}\left([0, T] ; C^{0}(\Gamma)\right)$ легко получить с учетом оценок $(2.8),(2.9)$ и равномерной ограниченности множества $M$ в этом пространстве.

Исследуем равностепенную непрерывность $B_{2} M$. Используя $(2.9)$, получаем

$$
\begin{aligned}
& \left\|\left(B_{2} h\right)\left(t_{2}, \cdot\right)-\left(B_{2} h\right)\left(t_{1}, \cdot\right)\right\|_{C^{0}(\Gamma)} \\
& \quad \leqslant\left\|\int_{t_{t}}^{t_{2}} \mathscr{B}_{2}\left(t_{2}-\tau\right) h(\tau) d \tau\right\|_{C^{0}(\Gamma)}+\left\|\int_{0}^{t_{1}}\left(\mathscr{B}_{2}\left(t_{2}-\tau\right)-\mathscr{B}_{2}\left(t_{1}-\tau\right)\right) h(\tau, \cdot) d \tau\right\|_{C^{0}(\Gamma)} \\
& \quad \leqslant c_{M} c_{1} T\left|t_{2}-t_{1}\right|+c_{M} \int_{0}^{T}\left|\mathscr{B}_{2}\left(t_{2}-\tau\right)-\mathscr{B}_{2}\left(t_{1}-\tau\right)\right| d \tau \\
& \quad \leqslant 2 c_{M} c_{1} T\left|t_{2}-t_{1}\right|, \quad h \in M, \quad t_{1}, t_{2} \in[0, T],
\end{aligned}
$$

где

$$
c_{1}=\frac{1}{2} \omega_{1}^{2}\left\|J_{1}(\cdot)(\cdot)^{-1}\right\|_{C^{0}[0, T]} \cdot
$$

По произвольному $\varepsilon>0$ выберем $\delta=\varepsilon /\left(2 c_{M} c_{1} T\right)$. Тогда если $\left|t_{2}-t_{1}\right|<\delta, t_{2}, t_{1} \in$ $[0, T]$, то

$$
\left\|\left(B_{2} h\right)\left(t_{2}, \cdot\right)-\left(B_{2} h\right)\left(t_{1}, \cdot\right)\right\|_{C^{0}(\Gamma)} \leqslant \varepsilon .
$$

В силу произвольности $\varepsilon>0$ множество $B_{2} M$ является равностепенно непрерывным, а оператор $B_{2}$ - компактньм.

Для исследования равностепенной непрерывности множества $B_{1} M$ заметим, что

$$
\begin{aligned}
\left(B_{1} h\right)\left(t_{2}, x\right)-\left(B_{1} h\right)\left(t_{1}, x\right) & =\int_{t_{1}}^{t_{2}} \frac{\partial}{\partial t}\left(B_{1} h\right)(t, x) d t \\
& =\int_{t_{1}}^{t_{2}} \int_{0}^{t} \int_{\Gamma} h(\tau, y) \frac{\partial}{\partial t} \mathscr{B}_{1}(t-\tau, x, y) d S_{y} d \tau d t
\end{aligned}
$$

Из явного выражения для $\mathscr{B}_{1}(t, x, y)$ и формулы $(2.3)$ получим оценку

$$
\left|\frac{\partial}{\partial t} \mathscr{B}_{1}(t-\tau, x, y)\right| \leqslant c_{0}\left[\frac{\left|\cos \left(x-y, \boldsymbol{n}_{y}\right)\right|}{4 \pi|x-y|^{2}}+1\right]
$$

где $c_{0}$ - некоторая константа. Согласно лемме 2 из $[15, \S 27.4]$ если $\Gamma \in C^{1, \lambda_{1}} \subset C^{2, \lambda}$, то существует такая константа $\widetilde{c}_{0}$, что сразу для всех $x \in \Gamma$ справедливо неравенство

$$
c_{0} \int_{\Gamma}\left[\frac{\left|\cos \left(x-y, \boldsymbol{n}_{y}\right)\right|}{4 \pi|x-y|^{2}}+1\right] d S_{y} \leqslant \widetilde{c}_{0} .
$$


Следовательно,

$$
\left\|\left(B_{1} h\right)\left(t_{2}, \cdot\right)-\left(B_{1} h\right)\left(t_{1}, \cdot\right)\right\|_{C^{0}(\Gamma)} \leqslant c_{M} \widetilde{c}_{0} T\left|t_{2}-t_{1}\right|
$$

Теперь по произвольному $\varepsilon>0$ выберем $\delta=\varepsilon /\left(c_{M} \widetilde{c}_{0} T\right)$. Тогда если $\left|t_{2}-t_{1}\right|<\delta$, $t_{2}, t_{1} \in[0, T]$, то

$$
\left\|\left(B_{1} h\right)\left(t_{2}, \cdot\right)-\left(B_{1} h\right)\left(t_{1}, \cdot\right)\right\|_{C^{0}(\Gamma)} \leqslant \varepsilon .
$$

В силу произвольности $\varepsilon$ множество $B_{1} M$ будет равностепенно непрерьвньг, а оператор $B_{1}$ - компактным. Из компактности операторов $B_{1}$ и $B_{2}$ следует справедливость леммы 9 .

\section{СПИСОК ЦИТИРОВАННОЙ ЛИТЕРАТУРЫ}

[1] Габов С. А., Шевцов П. В.Основные краевые задачи для уравнения колебаний стратифицированной жидкости // Докл. АН СССР. 1983. Т. 268. №6. С. 1293-1296.

[2] Габов С.А., Шевцов П. В. Об одном дифференциальном уравнении типа уравнения С. Л. Соболева // Докл. АН СССР. 1984. Т. 276. № 1. С. 14-17.

[3] Крутицкий П. А. Сведение второй начально-краевой задачи для уравнения гравитационно-гироскопических волн к однозначно-разрешимому интегральному уравнению // Дифференц. уравнения. 1996. Т. 32. № 10. С. 1386-1395.

[4] Успенский С. В., Демиденко Г. В., Перепелкин В. Г. Теоремы вложения и приложения к дифференциальным уравнениям. Новосибирск: Наука, 1984.

[5] Габов С.А., Свешников А.Г. Линейные задачи теории нестационарных внутренних волн. М.: Наука, 1990.

[6] Капитонов Б. В. Теория потенциала для уравнения малых колебаний вращающейся жидкости // Матем. сб. 1979. Т. 109. №4. С. 607-628.

[7] Крутицкий П. А. Явное решение задачи Дирихле для уравнения составного типа в многосвязной области // Докл. РАН. 1992. Т. 325. № 3. С. 426-431.

[8] Крутицкий П.А. Об одной начально-краевой задаче, описывающей колебания системы пластин в стратифицированной вращающейся жидкости // Математический анализ и дифференциальные уравнения. (сб. трудов). Новосибирск: Новосиб. Гос. ун-т, 1991. С. 73-88.

[9] Krutitskii P. A. An explicit solution of the pseudohyperbolic initial boundary value problem in a multiply connected region // Math. Meth. Appl. Sci. 1995. V. 18. № 11. P. 897-925.

[10] Крутицкий П. А. Первая начально-краевая задача для уравнения гравитационно-гироскопических волн в многосвязной области // ЖКВМиМФ. 1997. Т. 37. № 1. С. 117-128.

[11] Крутицкий П. А. Внешняя начально-краевая задача для одного эволюционного уравнения в многосвязной области // Дифференц. уравнения. 1998. Т. 34. № 12. С. 1624-1634.

[12] Крутицкий П. А. Вторая начально-краевая задача для уравнения гравитационно-гироскопических волн во внешней области // Матем. заметки. 1996. Т. 60. №1. С. 40-57.

[13] Бреховских А. М., Гончаров В.В.Введение в механику сплошных сред. М.: Наука, 1982.

[14] Le Blond P. H., Mysak L. A. Waves in the Ocean. Amsterdam: Elsevier, 1978.

[15] Владимиров В. С. Уравнения математической физики. М.: Наука, 1981.

[16] Соболев С. Л. Избранные вопросы теории функциональных пространств и обобщенных функций. М.: Наука, 1989.

[17] Колтон Д., Кресс Р. Методы интегральных уравнений в теории рассеяния. М.: Мир, 1987.

[18] Крейн С. Г. Функциональный анализ. М.: Наука, 1964.

[19] Ильин В. А., Позняк Э. Г. Основы математического анализа. Ч. 2. М.: Наука, 1973. 